\title{
On the Existence of Certain Smooth Toric Varieties*
}

\author{
Jörg Gretenkort, ${ }^{1}$ Peter Kleinschmidt, ${ }^{2}$ and Bernd Sturmfels ${ }^{3}$ \\ 'Institut für Ökonometrie und Operations Research, Universität Bonn, \\ Nassestrasse 2, 5300 Bonn, Federal Republic of Germany \\ ${ }^{2}$ Fakultät für Mathematik und Informatik, Universität Passau, \\ Innstrasse 27, 8390 Passau, Federal Republic of Germany \\ ${ }^{3}$ Institute for Mathematics and Its Applications, 514 Vincent Hall, 206 Church St. S.E., \\ University of Minnesota, Minneapolis, MN 55455, USA
}

\begin{abstract}
We prove that the combinatorial types of those cone systems which correspond to complete smooth toric varieties are more restricted than for complete toric varieties: the toric varieties corresponding to essentially all types of cyclic polytopes possess singularities. This yields a negative answer to a problem stated by $\mathrm{G}$. Ewald. Some consequences and problems concerning mathematical programming and the rational cohomology of smooth toric varieties are discussed.
\end{abstract}

\section{Introduction and Basic Terminology}

Recently, there has been much interaction between the combinatorial theory of polytopes and the theory of toric varieties [1], [3], [8], [9], [11]. A toric d-variety is a $T$-invariant subvariety of a $T$-invariant completion of the torus $T=\left(k^{*}\right)^{d}(k$ can be $\mathbb{C}$ or any algebraically closed field). It can be conveniently described in terms of a fan, i.e., a finite system of cones in $\mathbb{R}^{d}$ spanned by integer lattice points. In this paper we do not explain the numerous correspondences between properties of fans and properties of toric varieties. The interested reader should consult [2] and [9] for details, Here, we concentrate on those fans that correspond to complete smooth toric $d$-varieties.

Let $\Delta$ be a simplicial $(d-1)$-complex with the following properties:

(1) $\Delta$ is embedded in $\mathbb{R}^{d}$.

* The research of $\mathbf{P}$. Kleinschmidt was supported in part by the Institute for Mathematics and Its Applications, University of Minnesota, Minneapolis, Minnesota, USA. 
(2) The underlying set of $\Delta$, denoted $|\Delta|$, is homeomorphic to the unit-sphere $S^{d-1}$.

(3) The bounded component of $\mathbb{R}^{d}$ whose boundary is $|\Delta|$ contains the origin in its interior and is star-shaped with respect to the origin.

(4) The 0 -cells (vertices) of $\Delta$ are primitive lattice points in $\mathbb{Z}^{d}$.

(5) Let $x_{1}, \ldots, x_{d}$ be the vertices of a $(d-1)$-cell of $\Delta$, then

$$
\left|\operatorname{det}\left(x_{1}, \ldots, x_{d}\right)\right|=1 \text {. }
$$

Let $\Sigma_{\Delta}$ be the system of those cones whose apex is the origin and whose bases are the cells of $\Delta$. We call $\Sigma_{\Delta}$ a complete regular fan. In the absence of property (5) we call it a complete fan.

It is well known that all complete smooth toric $d$-varieties can be described in terms of complete regular fans [2] (smoothness is equivalent to condition (5)). Let $X_{\Sigma}$ denote the variety corresponding to the fan $\Sigma$. Two fans $\Sigma$ and $\Sigma^{\prime}$ are combinatorially equivalent (or isomorphic) if the complexes $\Delta$ and $\Delta^{\prime}$ which define $\Sigma$ and $\Sigma^{\prime}$ are combinatorially equivalent, i.e., there is a bijective inclusion preserving map from $\Delta$ to $\Delta^{\prime}$.

The varieties $X_{\Sigma}$ and $X_{\Sigma}$ are isomorphic if and only if such a combinatorial equivalence for $\Sigma$ and $\Sigma^{\prime}$ can be induced by a unimodular transformation of $\mathbb{R}^{d}$. So, the study of toric varieties can be viewed as the study of invariants of fans under unimodular transformations.

At the Fourth Geometry Symposium in Siegen, Ewald [4] posed the following question: Let $\Delta$ be a simplicial $(d-1)$-dimensional spherical complex. Then, does there exist a spherical complex $\Delta^{\prime}$ combinatorially equivalent to $\Delta$ such that $\Delta^{\prime}$ determines a complete regular fan?

This question was answered positively in [8] for the case that the number of vertices of $\Delta$ does not exceed $d+2$. In the present paper we give an infinite family of counterexamples to this question. The smallest complex in our family has $d+3$ vertices and is therefore minimal with the desired property.

Theorem. Let $\Delta$ be a complex which is isomorphic to the boundary-complex of the cyclic 4-polytope with $n \geq 7$ vertices (see [5] for the definition of cyclic polytopes). Then there does not exist a complex $\Delta^{\prime}$ with the properties (1)-(5) which is combinatorially equivalent to $\Delta$.

This theorem implies that no complete smooth toric 4-variety has an underlying cone-system stemming from a cyclic 4-polytope with more than six vertices. Hence, the existence of certain smooth toric varieties may be ruled out on grounds of the combinatorial type of a fan alone. In contrast to this it is well known [11] that every combinatorial type of a simplicial polytope gives rise to (many) complete fans which correspond to projective toric varieties. However, our result shows that under the regularity assumption this may not hold.

The proof of the theorem proceeds in two steps. In Section 2 we establish the case $n=7$, and in Section 3 a different technique of proof is used to settle the case $n \geq 8$. Finally, in Section 4 we discuss some consequences and problems related to our result. 


\section{On the Cyclic 4-Polytope with Seven Vertices}

Let us assume that $\Delta$ is a complex which has properties (1)-(5) of the last section and which is isomorphic to the boundary complex of the cyclic 4-polytope with seven vertices. Let $1,2, \ldots, 7$ denote the vertices of $\Delta$. Then Table 1 lists the 14 tetrahedra which are the maximal cells of $\Delta$.

Let $i_{1}, \ldots, i_{4}$ be the vertices of one of the listed tetrahedra and let $i_{1}, i_{2}, i_{3}, i_{5}$ be the vertices of the unique tetrahedron in the list with which the former shares the vertices $i_{1}, i_{2}$, and $i_{3}$. Then it follows from (5) that the following linear relation holds:

$$
i_{4}+i_{5}+\sum_{j=1}^{3} \lambda_{j} i_{j}=0 \quad \text { (for some integers } \lambda_{j} \text { ) }
$$

As $\Delta$ has 28 triangles, each of which is contained in precisely two of the tetrahedra, there are exactly 28 linear relations of type (*). (In [8] and [9] extensive use of these relations has been made for the classification of toric varieties.) Interpreting the coefficients of the points $i_{1}, \ldots, i_{7}$ in a relation of type $(*)$ as vectors in $\mathbb{R}^{7}$ (we call those vectors linear dependencies), it follows that the linear span of the linear dependencies is only three-dimensional.

So, there is a lot of redundancy in the 28 relations of type $(*)$. Using just this fact, it is easy to check that every relation (*) is equal to one of the 14 relations listed by their coefficients in Table 2 . The symbols in the left column are the tetrahedra from which the coefficients of the relation in the respective row come. The numbers $\lambda_{1}, \lambda_{2}, \ldots, \lambda_{35}$ are undetermined coefficients.

Using the fact that the rows $A B, B D$, and $B M$ in Table 2 are linearly independent and hence span the space of all linear relations, we can deduce further relations between the various $\lambda_{i}$. This could be done systematically using affine Grassmann-Plücker relations. However, as we are satisfied when a contradictory relation is reached, we do not present all relations and details here. These can be easily checked.

From the representation of all relations as linear combinations of $A B, B D$, and $B M$ we obtain among many other relations the following:

$$
\begin{aligned}
& \lambda_{1} \cdot \lambda_{10}=0, \\
& \lambda_{2} \cdot \lambda_{6}=0, \\
& \lambda_{5} \cdot \lambda_{8}=0 .
\end{aligned}
$$

Table 1

\begin{tabular}{llll}
\hline$A=1245$ & $E=1347$ & $I=2356$ & $M=2467$ \\
$B=1246$ & $F=1356$ & $J=2357$ & $N=3467$ \\
$C=1256$ & $G=1357$ & $K=2367$ & \\
$D=1346$ & $H=1457$ & $L=2457$ & \\
\hline
\end{tabular}


Table 2

\begin{tabular}{lccccccc}
\hline & \multicolumn{7}{c}{ Vertices } \\
\cline { 2 - 8 } Tetrahedra & 1 & 2 & 3 & 4 & 5 & 6 & 7 \\
\hline $\boldsymbol{A} B$ & $\lambda_{1}$ & $\lambda_{2}$ & 0 & 1 & 1 & 1 & 0 \\
$A L$ & 1 & 1 & 0 & $\lambda_{3}$ & $\lambda_{4}$ & 0 & 1 \\
$B D$ & $\lambda_{5}$ & 1 & 1 & $\lambda_{6}$ & 0 & $\lambda_{7}$ & 0 \\
$B M$ & 1 & $\lambda_{8}$ & 0 & $\lambda_{9}$ & 0 & $\lambda_{10}$ & 1 \\
$C F$ & 1 & 1 & 1 & 0 & $\lambda_{14}$ & $\lambda_{12}$ & 0 \\
$D E$ & 1 & 0 & $\lambda_{13}$ & $\lambda_{14}$ & 0 & 1 & 1 \\
$D F$ & $\lambda_{15}$ & 0 & $\lambda_{16}$ & 1 & 1 & $\lambda_{17}$ & 0 \\
$F G$ & $\lambda_{18}$ & 0 & $\lambda_{19}$ & 0 & $\lambda_{20}$ & 1 & 1 \\
$G H$ & $\lambda_{21}$ & 0 & 1 & 1 & 1 & 0 & $\lambda_{22}$ \\
$G J$ & 1 & 1 & $\lambda_{23}$ & 0 & $\lambda_{24}$ & 0 & $\lambda_{25}$ \\
$J K$ & 0 & $\lambda_{26}$ & $\lambda_{27}$ & 0 & 1 & 1 & 1 \\
$J L$ & 0 & $\lambda_{28}$ & 1 & 1 & $\lambda_{29}$ & 0 & $\lambda_{30}$ \\
$K N$ & 0 & 1 & 1 & 1 & 0 & $\lambda_{31}$ & $\lambda_{32}$ \\
$L M$ & 0 & $\lambda_{33}$ & 0 & $\lambda_{34}$ & 1 & 1 & $\lambda_{35}$ \\
\hline
\end{tabular}

As in each relation at least one of the $\lambda_{i}$ is equal to zero, we may branch the determination of all $\lambda_{i}$ into eight cases where three of the $\lambda_{i}$ from (1), (2), and (3) are set to zero. Each of these cases yields a contradiction for some of the $\lambda_{i}$. For example, if $\lambda_{1}=\lambda_{2}=\lambda_{5}=0$, it follows (from the additional relation $\lambda_{5}-\lambda_{1} \lambda_{6}=$ 1) that $1=0$. This proves the case $n=7$ of the theorem.

\section{On Cyclic 4-Polytopes with Eight or more Vertices}

In this section we complete the proof of the theorem by showing that no cyclic 4-polytope with eight or more vertices gives rise to a smooth toric variety. Let $\Delta$ be the boundary complex of a cyclic 4-polytope with $n \geq 8$ vertices, and assume that $\Delta$ is embedded with vertices $x_{1}, x_{2}, \ldots, x_{n}$ in $\mathbb{R}^{4}$ such that the properties (1) $-(5)$ in Section 1 are satisfied.

Every facet $\{i, j, k, l\}$ of $\Delta$ corresponds to a basis $\left\{x_{i}, x_{j}, x_{k}, x_{l}\right\}$ of $\mathbb{R}^{4}$, and the orientations of all such ordered bases induce an orientation of the simplicial complex $\Delta$. The 3-sphere $\Delta$ being closed, connected, and orientable, there exists (up to inversion) a unique such intrinsic orientation $\operatorname{sign}_{\Delta}$. This implies the following stronger version of property (5).

$\left(5^{\prime}\right)$ Let $x_{i}, x_{j}, x_{k}, x_{i}$ be the vertices of a tetrahedron of $\Delta$, then $[i, j, k, l]=$ $\operatorname{sign}_{\Delta}(i, j, k, l)$.

Here and throughout this section we use the abbreviation $[i, j, k, l]:=$ $\operatorname{det}\left(x_{i}, x_{j}, x_{k}, x_{l}\right)$.

Consider the simplicial $3-$ complex $\tilde{\Delta}$ on $\{1,2, \ldots, 8\}$ which is defined by the following set of 15 tetrahedra:

$$
\{\{r, r+1, r+s, r+s+1\} \mid 1 \leq r \leq 5,2 \leq s \leq 7-r\}
$$


By Gale's evenness condition [5], $\tilde{\Delta}$ is a subcomplex of $\Delta$, and it is easy to check that $\operatorname{sign}_{\Delta}(r, r+1, r+s, r+s+1)=+1$ for all $1 \leq r \leq 5$ and $2 \leq s \leq 7-r$. Observe that $\tilde{\Delta}$ is a 3 -ball, and therefore the unique orientation $\operatorname{sign}_{\tilde{\Delta}}$ of $\tilde{\Delta}$ is induced from $\operatorname{sign}_{\Delta}$.

Hence condition $\left(5^{\prime}\right)$ implies

$\left(5^{\prime \prime}\right)[r, r+1, r+s, r+s+1]=+1$ for all $1 \leq r \leq 5$ and $2 \leq s \leq 7-r$.

We will show that there do not exist vectors $x_{1}, x_{2}, \ldots, x_{8} \in \mathbb{R}^{4}$ satisfying the 15 determinant equations given in $\left(5^{\prime \prime}\right)$. Note that the corresponding system of equations has a solution for $n=7$, and hence the proof of the theorem had to split into two parts.

Following the general philosophy of "computational synthetic geometry" as outlined in [12], we give a compactly encoded nonrealizability proof for the synthetic geometry problem $\left(5^{\prime \prime}\right)$. The set $\{2,3,6,7\}$ being a tetrahedron of $\tilde{\Delta}$, it can be assumed that $\left(x_{2}, x_{3}, x_{6}, x_{7}\right)$ is a (positively oriented) orthonormal basis of $\mathbb{R}^{4}$, and we can write

$$
\left(x_{1}, x_{2}, \ldots, x_{8}\right)=\left(\begin{array}{cccccccc}
a & 1 & 0 & e & i & 0 & 0 & m \\
b & 0 & 1 & f & j & 0 & 0 & n \\
c & 0 & 0 & g & k & 1 & 0 & o \\
d & 0 & 0 & h & l & 0 & 1 & p
\end{array}\right),
$$

where $a, b, c, \ldots, o, p$ are indeterminates. Let

$$
\begin{array}{rrrrr}
P_{1}:=1-[1234], & P_{2}:=1-[1245], & P_{3}:=1-[1256], & P_{4}:=1-[1267], \\
P_{5}:=1-[1278], & P_{6}:=1-[2345], & P_{7}:=1-[2356], & P_{8}:=1-[2367], \\
P_{9}:=1-[2378], & P_{10}:=1-[3456], & P_{11}:=1-[3467], & P_{12}:=1-[3478], \\
P_{13}:=1-[4567], & P_{14}:=1-[4578], & P_{15}:=1-[5678], &
\end{array}
$$

For example, we have $P_{10}=1-e l+h i$.

Let $I$ denote the ideal in $\mathbb{Q}[a, b, \ldots, p]$ generated by the polynomials $P_{1}, P_{2}, \ldots, P_{1}$. It is clearly sufficient to show that $I$ has no complex zeros. To prove this constructively, we shall give a final polynomial [12, Chapter 4] for the realizability problem in question, that is, we shall construct polynomials $Q_{1}, \ldots, Q_{15} \in \mathbb{Q}[a, b, \ldots, p]$ such that $\sum_{i=1}^{15} Q_{i} P_{i}=1$.

Consider the elements $R_{1}$ and $R_{2}$ of $I$ which are defined as $R_{1}:=e P_{7}+P_{10}-P_{11}$, and

$$
\begin{aligned}
R_{2}:= & -n(c+k+i c f) P_{1}+i n P_{2}+(c n+k n+i g n) P_{3}+(o+i n h k+n c l+n k l) P_{4} \\
& +P_{5}-(m+2 i n-n c d-n k d-n c d i f+i f m+m f k) P_{6} \\
& -(n k+n c+m g+2 i n g-n i c f-n d g(c+k+i c f) \\
& +f m g(i+k)-c f e-e n k) P_{7} \\
& +(e-1+f(i+i e+k e)) P_{9}+(c f+n k) P_{10} \\
& -(1+j-j m+f(k+c+i)+n j d(c+k)) P_{11} \\
& +(1+f i+f k+j) P_{12}+(m-1-n c d-n k d) P_{13}+P_{14}-P_{15} .
\end{aligned}
$$


It can be seen that $R_{1}=i h$, and that $R_{2}+1$ is divisible by $h$. Let $S_{2}:=\left(R_{2}+1\right) / h \in$ $\mathbf{Q}[a, b, \ldots, p]$.

The combinatorial symmetry $\sigma=(18)(27)(36)(45)$ of $\tilde{\Delta}$ induces the symmetry $\tau=(a p)(b o)(c n)(d m)(e l)(f k)(g j)(h i)$ on the set $\left\{P_{1}, \ldots, P_{15}\right\}$ of generators of $I$. Applying the symmetry $\tau$ to the above representation of $R_{2}$ we get a representation of $R_{3}:=\tau\left(R_{2}\right)$ as a linear combination of the $P_{i}$. Clearly, $R_{3}+1$ is divisible by $i=\tau(h)$, and we can set $S_{3}:=\left(R_{3}+1\right) / i \in \mathbb{Q}[a, b, \ldots, p]$. Finally, observe the identity

$$
S_{2} S_{3} R_{1}-R_{2}-h S_{2} R_{3}=1
$$

This proves that $1 \in I$, and, moreover, with the above representations of $R_{1}, R_{2}$, and $R_{3}$, we immediately obtain polynomials $Q_{i}$ satisfying the desired identity $\sum_{i=1}^{15} Q_{i} P_{i}=1$.

\section{Consequences and Problems}

Let $X_{\Sigma}$ be a complete toric $d$-variety given by a fan $\Sigma$ or, equivalently, by a spherical complex $\Delta$ with $n$ vertices. Let $f_{i}$ be the number of $i$-dimensional cells of $\Delta$ and, for $0 \leq i \leq d$, let $h_{i}$ denote the following quantities:

$$
h_{i}:=\sum_{j=0}^{i}\left(\begin{array}{l}
d-j \\
d-i
\end{array}\right)(-1)^{i-j} f_{j-1} \quad\left(f_{-1}:=1\right) .
$$

It is well known [10] that these $h_{i}$ count the cocycles which generate the rational cohomology of $X_{\Sigma}$. More precisely, $h_{i}=\mathrm{rk} H^{2 i}\left(X_{\Sigma}, \mathbb{Q}\right), 0 \leq i \leq d$ (note that the odd-dimensional cohomology vanishes).

As $|\Delta|$ is a sphere, the upper-bound-theorem for spheres (UBTS for short) [10] says that

$$
\begin{aligned}
& h_{i} \leq\left(\begin{array}{c}
n-d-1 \\
i
\end{array}\right), \quad \text { or } \quad\left(\text { as } h_{1}=n-d\right) \\
& h_{i} \leq\left(\begin{array}{c}
h_{1}+i-1 \\
i
\end{array}\right) \quad \text { for } 0 \leq i \leq\left[\frac{d}{2}\right] .
\end{aligned}
$$

The Dehn-Sommerville equations [5] are equivalent to $h_{i}=h_{d-i}, 0 \leq i \leq d$, so that the above bounds for the $h_{i}$ imply bounds for the other $h_{i}$ as well. All these bounds are simultaneously achieved if $\Delta$ is isomorphic to the boundary complex of the cyclic $d$-polytope with $n$ vertices.

As mentioned before, it is well known that for every combinatorial type of a simplicial $d$-polytope there is a complete (even projective) toric $d$-variety whose fan is given by a complex which is isomorphic to the boundary complex of that polytope. So, the above bounds for the $h_{i}$ are also sharp upper bounds for the ranks of rational cohomology of complete toric $d$-varieties. So, UBTS has the following counterpart for toric varieties (call it UBTV for short): 
Let $X$ be a complete toric $d$-variety with rk $H^{2}(X, Q)=: k$ fixed. Then the following holds:

$$
\text { rk } H^{2 i}(X, \mathbb{Q}) \leq\left(\begin{array}{c}
k+i-1 \\
i
\end{array}\right), \quad 0 \leq i \leq\left[\frac{d}{2}\right],
$$

and the bounds are achieved by fans which are given by the boundary complexes of cyclic $d$-polytopes with $k+d$ vertices. So the number $k$ already determines sharp upper bounds for the number of higher-dimensional cohomology classes.

It is a consequence of our theorem in Section 1 that the statement about sharpness of the UBTV is not valid for smooth $X$ with $d=4$ and $k=3$. For the combinatorial type of a corresponding sphere with $h_{2}=6$ (for sharpness) would have to be one of the cyclic 4-polytope with seven vertices [5]. Our theorem, however, implies that this type does not give rise to a smooth toric variety. So we have (in contrast to the UBTV):

Corollary 1. Let $X$ be a complete smooth toric 4 -variety with $\mathrm{rk} H^{2}(X, \mathbb{Q})=3$. Then rk $H^{4}(X, \mathbb{Q}) \leq 5$, This bound can be achieved.

This corollary is an immediate consequence of our theorem and the UBTS. The statement about sharpness follows by blowing-up a suitable 4-variety from [8]. It is not surprising that smoothness implies fewer cocycles. However, we are not aware of any other result which directly implies Corollary 1.

It would be of interest to prove a correct version of the UBTV for the smooth case, i.e., to determine sharp upper bounds for rk $H^{2 i}(X, \mathbb{Q})$ given rk $H^{2}(X, \mathbb{Q})$ for complete smooth toric $d$-varieties. It may be guessed that asymptotically these bounds are far smaller than those given in the UBTV. A result of this type would allow us to rule out smoothness of a variety by computing some of the $h_{i}$.

Such bounds would be of interest in mathematical programming. We can interpret the cones of a complete fan as the cones spanned by the normals of the hyperplanes which determine a basic feasible solution of a nondegenerate LP [7]. The regularity condition is fulfilled if the LP-matrix is totally unimodular (or if each maximal quadratic submatrix belonging to a feasible basis has determinant \pm 1 ). So, such a version of a UBTV would yield sharp upper bounds for the number of basic feasible solutions of such LPs.

It is well known [5] that for $d \geq 3$ every cyclic $d$-polytope with $v$ vertices has a vertex whose vertex figure (or link) is isomorphic to the boundary complex of a cyclic $(d-1)$-polytope with $v-1$ vertices. It follows from this fact and the results of [9] that a smooth toric $d$-variety arising from a complete regular fan which is isomorphic to the boundary-complex of a cyclic $d$-polytope with $v$ vertices possesses a smooth toric $(d-1)$-subvariety which comes from a complete regular fan which is isomorphic to the boundary-complex of a cyclic $(d-1)$-polytope with $v-1$ vertices. This fact and our theorem directly imply:

Corollary 2. Let $\Delta$ be a complex which is isomorphic to the boundary-complex of the cyclic $d$-polytope with $n$ vertices, $n \geq d+3 \geq 7$. Then there does not exist a complex $\Delta^{\prime}$ with the properties (1)-(5) which is combinatorially equivalent to $\Delta$. 
This proves that for $n \geq d+3 \geq 7$, no complete smooth toric $d$-variety has an underlying cone-system stemming from a cyclic $d$-polytope with $n$ vertices.

Applying the methods of Section 3 to the three noncyclic neighborly 3-spheres with eight vertices given in the list of Grünbaum and Sreedharan [6], we were able to extend Corollary 1 from seven to eight vertices. Note that among these three spheres only two are boundary complexes of 4-polytopes with eight vertices ( $P_{35}^{8}$ and $P_{36}^{8}$ in [6]) while the third one is the nonpolytopal Brückner sphere (denoted $\mathcal{M}$ in [6]). More precisely, we have:

Remark. Let $X$ be a complete toric 4-variety with $\mathrm{rk} H^{2}(X, \mathbb{Q})=4$. Then rk $H^{4}(X, \mathbb{Q}) \leq 10$, and this bound is attained by four combinatorial types of fans. If, in addition, $X$ is assumed to be smooth, then we have rk $H^{4}(X, \mathbb{Q}) \leq 9$.

In view of these results we conjecture that, for $d \geq 4$, no neighborly $d$-polytope with $d+3$ or more vertices gives rise to a complete smooth toric $d$-variety.

\section{References}

1. V. V. Batyrev, Toroidal Fano 3-folds, Math. USSR-Izv. 19 (1982), 13-25.

2. V. I. Danilov, The geometry of toric varieties, Math. USSR-Izv. 17 (1981), 97-154.

3. G. Ewald, Spherical complexes and nonprojective toric varieties, Discrete Comput. Geom. 1 (1986), 115-122.

4. G. Ewald, Unsolved problems in combinatorial convexity relevant for algebraic geometry, Abstracts of the 4th Geometry Symposium in Siegen (1986).

5. B. Grünbaum, Convex Polytopes, Interscience, New York, 1967.

6. B. Grünbaum and V.P. Sreedharan, An enumeration of simplicial 4-polytopes with eight vertices, J. Combin. Theory 2 (1967), 437-465.

7. V. L. Klee and P. Kleinschmidt, Geometry of the Gass-Saaty parametric LP algorithm, Discrete Comput. Geom. (to appear).

8. P. Kleinschmidt, A classification of toric varieties with few generators, Aequationes Math. (to appear).

9. T. Oda, Torus Embeddings and Applications, Tata Institute of Fundamental Research Lecture on Mathematics and Physics, Vol. 57, Springer-Verlag, Berlin, 1978.

10. R. P. Stanley, The upper bound conjecture and Cohen-Macaulay-rings, Stud. Appl. Math. 54 (1975), 135-142.

11. R. P. Stanley, The number of faces of a simplicial convex polytope, Adv. in Math. 45 (1980), 236-238.

12. B. Sturmfels, Computational Synthetic Geometry, Ph.D. Dissertation, University of Washington, 1987.

Received April 1, 1987, and in revised form October 22, 1987. 